\title{
Forehead galeal pericranial flap for single-staged total upper eyelid reconstruction in sebaceous gland carcinoma excision
}

This article was published in the following Dove Press journal:

International Medical Case Reports Journal

5 September 2017

Number of times this article has been viewed

\author{
Hui-hsiu Chang ${ }^{1,2}$ \\ Edward Suh ${ }^{3}$ \\ Blake H Fortes ${ }^{3}$ \\ Franklin Zheng ${ }^{3}$ \\ Anny MS Cheng ${ }^{3,4}$
}

'Division of Plastic and

Reconstructive Surgery, Department of Surgery, National Taiwan University Hosiptal Yunlin Branch, Yunlin, Taiwan; ${ }^{2}$ Department of Industrial Engineering and Management, National Yunlin University of Science and Technology, Yunlin, Taiwan; ${ }^{3}$ Department of Ophthalmology, Florida International University, Herbert Wertheim College of Medicine, Miami, FL, USA; ${ }^{4}$ Ocular Surface Center, Miami, FL, USA
Correspondence: Anny MS Cheng Ocular Surface Center, 7000 S.W. 97th Avenue, Suite 213, Miami, FL 33173, USA Tel +l 3052741299

Fax + I 305274 I297

Email acheng@fiu.edu
Purpose: To present a successful case of single-staged total upper eyelid reconstruction after sebaceous gland carcinoma excision by using forehead galeal pericranial flap.

Observations: An 80-year-old female with a progressively enlarged left upper eyelid mass presented with ocular irritation, blurred vision, and gritty sensation despite topical antibiotics treatment. This multinodular mass involved the left total upper eyelid, compromised corneal surface integrity, and caused complete ptosis. Excisional biopsy confirmed advanced sebaceous gland carcinoma, which was followed by extensive excision. The resultant total upper eyelid defect was reconstructed by a forehead galeal pericranial flap accompanied by anterior and posterior lamellar grafts. For the 34-month follow-up period, patient remained symptom-free without tumor recurrence and achieved acceptable cosmetic outcome.

Conclusion: The forehead galeal pericranial flap appears to be effective as single-staged total upper lid reconstruction following extensive sebaceous gland carcinoma excision to restore eyelid function, avoid corneal exposure, and achieve acceptable cosmesis.

Keywords: forehead, galeal pericranial flap, reconstruction, sebaceous gland carcinoma, singlestaged, total upper eyelid reconstruction

\section{Introduction}

Total eyelid defect caused by the loss of the full thickness of the eyelids from medial to lateral canthus commonly results from periocular tumor excision. Although rare but easily overlooked, sebaceous gland carcinoma is a highly malignant eyelid tumor that often results in extensive tumor excision. ${ }^{1}$

It remains challenging to reconstruct the aforementioned total upper eyelid defect. Two-staged surgical treatment techniques, including Cutler-Beard, ${ }^{2}$ Mustardè, ${ }^{3}$ modified Hughes, ${ }^{4}$ tarsoconjunctival rotational, ${ }^{5}$ or advancement, ${ }^{6}$ have been described. To reduce surgical sessions and decrease eyelid donor tissue morbidity, single-staged surgery utilizing frontalis muscle flap ${ }^{7,8}$ and forehead galeal pericranial flap have demonstrated promising results. Forehead galeal pericranial flap has been employed during medial canthus reconstruction, ${ }^{9}$ subtotal and total upper lid reconstruction for tumor excision, and periorbital faciitis, ${ }^{10}$ respectively. The galeal aponeurotica consists of dense connective tissue that originates on the occipital bone and inserts onto the frontalis muscle. These nonbulky flaps are viable to ensure good functional and morphological outcomes, avoid a second surgical session, preserve local tissues and innervations, and achieve satisfactory cosmesis. Herein, we reported a single case demonstrating how this procedure can be used as an effective reconstruction option for advanced sebaceous carcinoma following total upper lid excision. 


\section{Case report}

An 80-year-old female complained of a 1-year history of ocular irritation, blurred vision, and gritty sensation despite topical tetracycline $1 \%$ three times daily and erythromycin $0.5 \%$ twice a day for refractory blepharoconjunctivitis and recurrent chalazion in the left eye. On examination, her bestcorrected visual acuity was 20/20 in the right eye and hand motion in the left eye. External examination revealed mild telangiectasia on the left upper eyelid with a mass consisting of multiple, firm, thickened, irregular nodules (Figure 1). This mass was fixed to the underlying tissue and resulted in complete ptosis without any eyelid blinking. Consequently, the left eye exhibited a scanty tear meniscus, central corneal haze and neovascularization, and diffuse conjunctival injection.

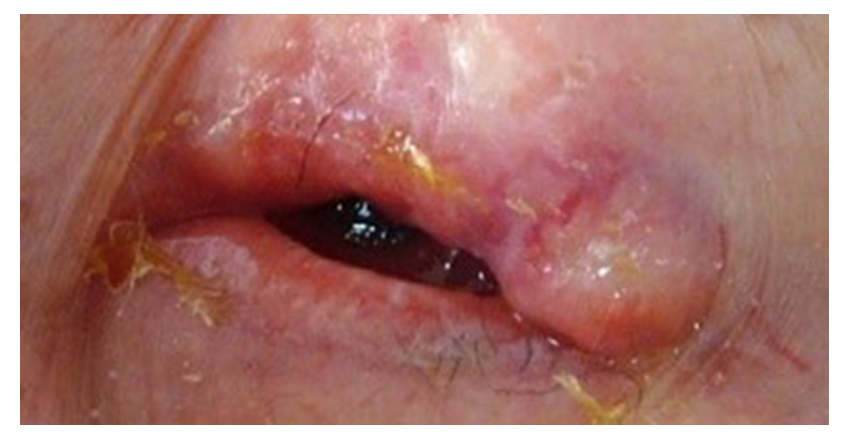

Figure I An extensive left upper eyelid mass.

Notes: The elevated, firm, irregular multinodular mass involved left total upper eyelid, compromised the corneal surface integrity, and was associated with complete ptosis.
We obtained written informed consent from the patient to perform the following surgical procedures as well as consent to the publication of her case details and any accompanying images. We first performed incisional wedge of the left upper eyelid. Microscopic examination disclosed a poorly differentiated sebaceous carcinoma with infiltrative growth and pagetoid invasion of overlying eyelid epithelium and deep superficial orbicularis muscle. Then under general anesthesia, we performed total removal of the tumor in the left upper lid including superior conjunctival fornix and nearly total removal of levator muscle resulting in the full-thickness eyelid defect measuring $4.5 \times 1.5 \mathrm{~cm}$, extending from the medial and lateral canthi to the inferior eyebrow (Figure 2A). This defect was reconstructed by a hard palate graft secured to the remnants of the conjunctiva with 6-0 Vicryl (Polyglactin 910, Ethicon ${ }^{\circledR}$, Somerville, NJ, USA) at the resection borders as posterior lamella. Then we obtained the galeal pericranial flap measuring $4 \times 4.5$ $\mathrm{cm}$ by an incision along the frontal skin crease above the eyebrow through the skin, and subcutaneous tissues, dissection above the frontalis muscle, and vertical relaxing incisions medially and laterally (Figure 2B, illustrated as 2C [coronal plane] or 2D [sagittal plane]). Blunt dissection along the soft tissues of the eyebrow and supraorbital margin was performed to create a tunnel to flip and slide the galeal pericranial flap to reach the previous upper lid
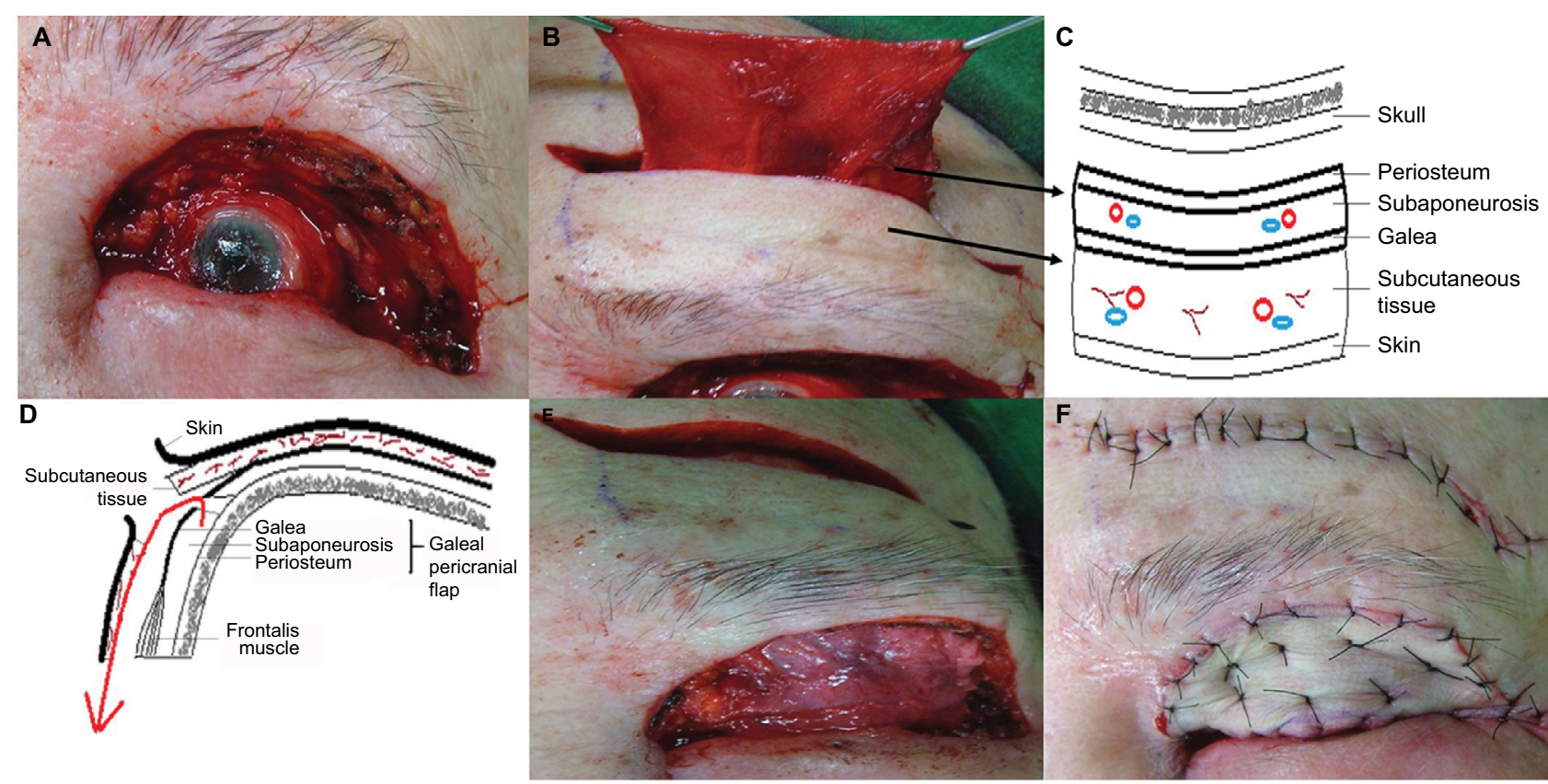

Figure 2 Surgical technique for single-staged total upper eyelid reconstruction after sebaceous gland carcinoma excision.

Notes: The tumor was extensively removed by excision of the full-thickness eyelid with extension to inferior eyebrow, and medial and lateral canthi (A). A hard palate graft was secured to the remnants of the conjunctiva at the resection eyelid borders as posterior lamella. The isolated forehead galeal pericranial flap (B) was used as a tough supportive connective tissue (C, coronal or $\mathbf{D}$, sagittal planes) to suture the remnant of the resected upper eyelid borders (E) and stump of the levator aponeurosis. Finally, a full-thickness skin graft overlying galeal pericranial flap was harvested to reconstruct the anterior lamella (F). 
margin. Because the levator muscle was nearly completely removed as a result of extensive tumor involvement, we were able to identify the remaining stump of the elevator aponeurosis and reanchor it to the flap to preserve some levator function. The upper margin of the galeal pericranial flap was sutured with $6-0$ Vicryl to the remnant of the resected medial and lateral left upper eyelid borders (Figure 2E). Finally, a full-thickness skin graft was harvested from the patient's inguinal area to cover the external surface of the galeal pericranial flap to reconstruct the anterior lamella (Figure 2F).

One month after surgery, ocular symptoms completely resolved and the flaps and grafts were viable with no evidence of necrosis. Partial forehead sensation remained intact and there was preservation of muscle tone. Patient had acceptable eyelid closure (Figure 3A) and regained 3-4 mm eyelid opening (Figure 3B). Two months later, she developed tumor metastasis to the ipsilateral parotid gland lymph nodes and subsequently received adjuvant chemotherapy and local radiation therapy. Another month later, her left eye showed regressed corneal neovascularization, uninflamed conjunctiva, moderate tear meniscus, and improved visual acuity from hand motion to 20/400. No postoperative complications such as lid retraction, corneal erosion, or ulceration were observed. For a total follow-up period of 34 months, she remained symptom free without eyelid tumor recurrence and achieved acceptable cosmetic outcome.

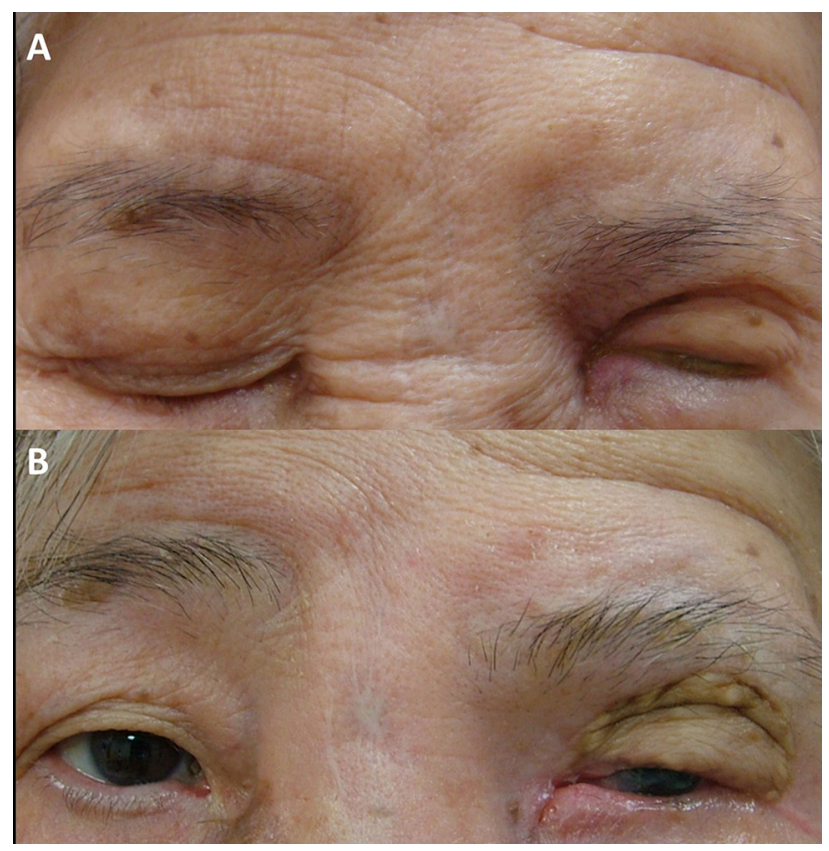

Figure 3 Surgical outcome.

Notes: Ocular symptoms completely resolved and the flaps and grafts were viable. Patient had acceptable eye lid closure (A) and 3-4 mm eyelid opening (B) after the surgery.

\section{Discussion}

Despite the numerous traditional two-staged reconstructive surgical methods reported over the years, ${ }^{2-6}$ total upper eyelid restoration remains complex and challenging owing to the limited neighboring tissue as well as the difficulty in preserving the highly specialized function of the upper eyelid. Although a two-staged procedure may be valuable, single-staged reconstructive surgery has gained considerable interest for simplifying procedures by avoiding lower lid disruption, minimizing donor tissue morbidity, and has proven worthy of joining the treatment options to ensure an optimal outcome. In accordance with recent single-staged surgical developments, ${ }^{11-13}$ herein we present a case of extensive sebaceous gland carcinoma excision followed by successful single-staged total upper eyelid reconstruction with forehead galeal pericranial flap. After the surgery, patient experienced complete symptom resolution, as well as improved corneal integrity and visual acuity. Consistent with previous report ${ }^{10}$ this case highlights single-staged reconstructive surgery with galeal pericranial flap as an effective treatment to restore eyelid function, avoid corneal exposure, and achieve satisfactory cosmesis.

Harvesting the galeal pericranium without complete disruption of the frontalis muscle helps preserve forehead sensation and motility during facial expression. In addition, harvesting hard palate and inguinal skin has minimal donor-site morbidity. While the thickness and potential scarring of a frontalis muscle flap may be less satisfactory for upper lid reconstruction in one study ${ }^{14}$ but not others, ${ }^{7,8}$ the fibrous sheet of galeal pericranial flap in this case is ideal owing to the thin but remarkably tough connective tissue to support anterior and posterior lamella grafts. In addition, the galeal pericranial flap serves as a good vascular supply to both the underlying tarsal graft and the overlying free skin graft. ${ }^{15}$ Furthermore, the forehead horizontal wrinkle lines in the patient conceal the transverse incisions as the scar is hidden in the crease. Although the remaining levator muscle was nearly completely removed resulting in limited eyelid excursion, this technique avoids postoperative lid retraction or exposure keratopathy and allows reconstruction of not only large horizontal but also vertical defects. Collectively, the single-staged forehead galeal pericranial flap appears to be a reliable surgical option for total upper lid reconstruction.

\section{Acknowledgment}

The authors gratefully thank Dr. Scheffer C.G. Tseng for his guidance and great support.

\section{Disclosure}

The authors report no conflicts of interest in this work. 


\section{References}

1. Muqit MM, Foot B, Walters SJ, Mudhar HS, Roberts F, Rennie IG. Observational prospective cohort study of patients with newly-diagnosed ocular sebaceous carcinoma. Br J Ophthalmol. 2013;97(1):47-51.

2. Cutler NL, Beard C. A method for partial and total upper lid reconstruction. Am J Ophthalmol. 1955;39(1):1-7.

3. Mustarde JC. Major reconstruction of the eyelids: functional and aesthetic considerations. Clin Plast Surg. 1981;8(2):227-236.

4. Mauriello JA Jr, Antonacci R. Single tarsoconjunctival flap (lower eyelid) for upper eyelid reconstruction ("reverse" modified Hughes procedure). Ophthalmic Surg. 1994;25(6):374-378.

5. Kersten RC, Anderson RL, Tse DT, Weinstein GL. Tarsal rotational flap for upper eyelid reconstruction. Arch Ophthalmol. 1986;104(6):918-922.

6. Leone CR Jr. Tarsal-conjunctival advancement flaps for upper eyelid reconstruction. Arch Ophthalmol. 1983;101(6):945-948.

7. Stewart CM, Tan LT, Johnson D, Norris JH. Key Issues When reconstructing extensive upper eyelid defects with description of a dynamic, frontalis turnover flap. Ophthal Plast Reconstr Surg. 2016;32(4):249-251.

8. Jean RD, Wong WW, Martin MC. Single-stage frontalis muscle flap for full-thickness reconstruction of the upper eyelid. J Craniofac Surg. 2011;22(5):1762-1764.
9. Leatherbarrow B, Watson A, Wilcsek G. Use of the pericranial flap in medial canthal reconstruction: another application for this versatile flap. Ophthal Plast Reconstr Surg. 2006;22(6):414-419.

10. Brusati R, Colletti G, Redaelli V. Upper eyelid reconstruction with forehead galeal flap. J Plast Reconstr Aesthet Surg. 2009;62(7): 901-905.

11. Scuderi N, Ribuffo D, Chiummariello S. Total and subtotal upper eyelid reconstruction with the nasal chondromucosal flap: a 10-year experience. Plast Reconstr Surg. 2005;115(5):1259-1265.

12. Toft PB. Reconstruction of large upper eyelid defects with a free tarsal plate graft and a myocutaneous pedicle flap plus a free skin graft. Orbit. 2016;35(1):1-5.

13. Pushker N, Batra J, Meel R, Bajaj MS, Chawla B, Ghose S. Lateral eyelid rotation flap: a novel technique for reconstruction of full thickness eyelid defect. Int ophthalmol. 2015;35(6):793-799.

14. Kazanjian VH, Roopenian A. Median forehead flaps in the repair of defects of the nose and surrounding areas. Trans Am Acad Ophthalmol Otolaryngol. 1956;60(4):557-566.

15. Tse DT, Goodwin WJ, Johnson T, Gilberg S, Meldrum M. Use of galeal or pericranial flaps for reconstruction or orbital and eyelid defects. Arch Ophthalmol. 1997;115(7):932-937.
International Medical Case Reports Journal

\section{Publish your work in this journal}

The International Medical Case Reports Journal is an international, peer-reviewed open-access journal publishing original case reports from all medical specialties. Previously unpublished medical posters are also accepted relating to any area of clinical or preclinical science. Submissions should not normally exceed 2,000 words or
4 published pages including figures, diagrams and references. The manuscript management system is completely online and includes a very quick and fair peer-review system, which is all easy to use. Visit http://www.dovepress.com/testimonials.php to read real quotes from published authors. 